Research Article

\title{
Preparation and Characterization of Rigid Polyurethane Foams with Different Loadings of Lignin-Derived Polycarboxylic Acids
}

\author{
Ruiqi Wang $\mathbb{D}^{D}$, Bing Zhou $\mathbb{D}^{\text {D }}$, Yanchao Zhu $\mathbb{D}^{D}$, and Zichen Wang \\ College of Chemistry, Jilin University, Qianjin Street 2699, Changchun 130012, China \\ Correspondence should be addressed to Yanchao Zhu; yanchao_zhu@jlu.edu.cn
}

Received 14 December 2018; Revised 1 April 2019; Accepted 15 April 2019; Published 19 May 2019

Academic Editor: Domenico Acierno

Copyright (C) 2019 Ruiqi Wang et al. This is an open access article distributed under the Creative Commons Attribution License, which permits unrestricted use, distribution, and reproduction in any medium, provided the original work is properly cited.

Lignin was modified by oxidation to prepare lignin-derived polycarboxylic acids (LPCAs). LPCAs can be introduced into rigid polyurethane foams (RPUFs) via 1,4-dioxane. The expansion ratio and compressive strength of RPUFs were observed. When the loading of LPCAs was $0.67 \mathrm{wt} \%$ (based on polyol), the compressive strength of RPUF was the highest and was about $59.2 \%$ higher than that of the blank RPUF. The reinforcing mechanism of LPCAs was supposed that the interactions between LPCAs and RPUFs increased the strength of the cell walls. When the loading of LPCAs was less than 0.33 wt $\%$, the expansion ratio of RPUFs increased slightly. The water resistance of RPUFs was not affected by LPCAs, suggesting that RPUFs with LPCAs could be used in a humid environment. Besides, the application of the oxidative modification products of lignin was broadened.

\section{Introduction}

Rigid polyurethane foam (RPUF) is a widely used engineering material. Because of its outstanding performance of thermal insulation, low-density, and high-mechanical properties, it can serve as a thermal insulation material, potting material, load-bearing structure material, etc. It has been produced in a large number and applied in many aspects such as constructions, refrigerators, and automotive industry [1-3]. Conventionally, polyurethane foams are manufactured from petroleum-derived materials but they are hard to recycle in general, which has brought about considerable environment concerns. In recent years, rising petroleum prices and sustainability issues remind people to focus on biomass-derived materials. To decrease the use of fossil resources, many kinds of renewable sources were introduced into polyurethane foams $[4,5]$. For example, bio-based polyols were often used in synthesis of polyurethane foams to reduce the use of petroleum-derived polyols $[6,7]$.

Lignin, an important and abundant bioresource in nature, is the only natural polymer with aromatic structures [8]. Lignin is usually discarded with the pulping waste liquor or burned with biomass fuels, causing a waste of resources and a great deal of environment pollution problems. To prevent waste and low-value utilization, lignin is under growing considerations and researches [9]. Through different isolation processes, the original lignin can be transformed into various types like kraft lignin, lignosulfonate, organosolv lignin, and steam explosion lignin [10]. With several functional groups such as carboxyl, methoxyl, and hydroxyl [11], lignin can be chemically modified by different methods like hydroxymethylation, Mannich reaction, alkylation, and oxidation $[12,13]$. Therefore, lignin can be used to prepare bio-based polyols and serve as a polyol substitution in RPUFs, which can increase the degradability of RPUFs [14]. As a modification method of lignin, oxidation is also a promising way to obtain valuable products. Many reagents, like nitrobenzene, hydrogen peroxide, metal oxides, and laccase, have been used to oxidize lignin, and the corresponding applications have been achieved $[9,15,16]$. For instance, the solutions of oxidized lignin could be used as a ready-to-use concrete plasticizer [17]. Exploiting oxidative products of lignin contributes to develop more high-value applications of lignin [18]. 
In this work, lignin-derived polycarboxylic acids (LPCAs), the oxidation products of lignin, were introduced into RPUFs. $\mathrm{NaOCl}$ solution, an oxidant which can be utilized under mild conditions in labs, was selected to perform oxidative modification on steam explosion lignin to prepare LPCAs. The changes of the expansion ratio and compression properties of RPUFs with different loadings of LPCAs were observed. In addition, the water resistance of RPUFs was also studied.

\section{Materials and Methods}

2.1. Materials. Steam explosion lignin was donated by Jilin KAIYU Biomass Development and Utilization Co. Ltd. Methylene diphenyl diisocyanate (MDI) (model number: PM-200) was provided by Jining Baichuan Chemical Co. Ltd. Polyether polyol (model number: DV-125) was obtained from Shandong Bluestar Dongda Co. Ltd. Silicone-based surfactant (model number: AK-158) was supplied by Jining Hengtai Chemical Co. Ltd. All the other chemical reagents were purchased in China and were of analytical grade. The concentration of $\mathrm{NaOCl}$ solution was calibrated according to the China National Standard GB 19106-2003.

2.2. Preparation of LPCAs. $5.00 \mathrm{~g}$ steam explosion lignin and $150 \mathrm{~mL}$ distilled water were placed into a $500 \mathrm{~mL}$ three-neck round-bottom flask. The mixture in the flask was heated to $30^{\circ} \mathrm{C}$ in a water bath and was stirred mechanically. Then $\mathrm{NaOCl}$ solution was added at a ratio $(n(\mathrm{NaOCl}) / m($ lignin $))$ of $12 \mathrm{mmol} \cdot \mathrm{g}^{-1}$ in $10 \mathrm{~min}$. The total time of stirring was $30 \mathrm{~min}$. Afterwards, $6 \mathrm{~mol} \cdot \mathrm{L}^{-1} \mathrm{HCl}$ was added to achieve a $\mathrm{pH}$ of 2 (using $\mathrm{pH}$ test strips with a range of 1 to 14). The precipitate was centrifuged, washed once with distilled water, dried at $80^{\circ} \mathrm{C}$ for $24 \mathrm{~h}$, and ground to obtain LPCAs. To compare the effect of oxidative modification, some amount of acid-precipitated lignin was prepared from steam explosion lignin.

2.3. Synthesis of RPUFs. In order to introduce LPCAs into RPUFs, 1,4-dioxane (called "dioxane" for short in the following) was used to dissolve LPCAs, which could prevent LPCAs from becoming rigid particles and help LPCAs to modify RPUFs at the molecular level. Firstly, $0.02 \mathrm{~g}$ LPCAs was added into a test tube and then $0.70 \mathrm{~g}$ dioxane and $0.17 \mathrm{~g}$ distilled water were added successively to prepare the solution of LPCAs $(0.70 \mathrm{~g}$ dioxane and $0.17 \mathrm{~g}$ water made up $0.80 \mathrm{~mL}$ $80 \%(v / v)$ dioxane-water solution). Secondly, $15.00 \mathrm{~g}$ polyether polyol, $0.08 \mathrm{~g}$ dibutyltin dilaurate (DBTDL), $0.40 \mathrm{~g}$ surfactant, $0.58 \mathrm{~g}$ distilled water, and the solution of LPCAs prepared previously were placed into a $100 \mathrm{~mL}$ beaker to make up Solution A of RPUF. Solution A was stirred magnetically for $10 \mathrm{~min}$, and then it was transferred into a cylindrical mold, which was used for the foaming process. Next, $24.00 \mathrm{~g}$ MDI (Solution B) was added into the mold. The mixture in the mold was stirred mechanically for $30 \mathrm{~s}$, and later, it foamed in free space to produce RPUF. Besides, a blank bulk of RPUF was synthesized, in which both LPCAs and dioxane were not added.
TABLE 1: The amount of LPCAs, the loading of LPCAs, and the label of RPUFs.

\begin{tabular}{lcc}
\hline RPUF label & LPCA amount & LPCA loading \\
\hline F0 & $0 \mathrm{~g}$ & $0 \mathrm{wt} \%$ \\
F1 & $0.02 \mathrm{~g}$ & $0.13 \mathrm{wt} \%$ \\
F2 & $0.05 \mathrm{~g}$ & $0.33 \mathrm{wt} \%$ \\
F3 & $0.08 \mathrm{~g}$ & $0.53 \mathrm{wt} \%$ \\
F4 & $0.10 \mathrm{~g}$ & $0.67 \mathrm{wt} \%$ \\
F5 & $0.15 \mathrm{~g}$ & $1.00 \mathrm{wt} \%$ \\
\hline
\end{tabular}

The loading of LPCAs in RPUFs is expressed as the weight ratio of LPCAs vs polyol, i.e., $m$ (LPCAs) $/ m$ (polyol) $\times 100 \%$. For ease of expression, each RPUF is assigned with a label. The label of RPUFs, the amount of LPCAs, and the loading of LPCAs are shown in Table 1.

2.4. Characterization of LPCAs. The content of carboxyl was measured by titration analysis [19]. $200 \mathrm{mg}$ LPCAs and $20 \mathrm{~mL} 0.4 \mathrm{~mol} \cdot \mathrm{L}^{-1}$ calcium acetate solution were mixed in a $100 \mathrm{~mL}$ round-bottom flask. The mixture was heated at $85^{\circ} \mathrm{C}$ for $30 \mathrm{~min}$. Then it was cooled to room temperature and filtrated. The filtrate was transferred into a $50 \mathrm{~mL}$ volumetric flask and was diluted by distilled water to reach the graduation. $20 \mathrm{~mL}$ distilled water and $10 \mathrm{~mL}$ solution in the volumetric flask were added into a $250 \mathrm{~mL}$ conical flask. $0.05 \mathrm{~mol} \cdot \mathrm{L}^{-1} \mathrm{NaOH}$ solution was used in titration with phenolphthalein as the indicator. Blank test was performed meanwhile, in which the sample was not used. The content of carboxyl was calculated according to the following:

$$
w(\mathrm{COOH})=\frac{\left(V_{1}-V_{0}\right) \times c \times 5 \times 45.018}{m} \times 100 \%
$$

where $V_{1}$ and $V_{0}$ were the volume of $\mathrm{NaOH}$ standard solution that LPCAs and the blank experiment consumed, respectively, $c$ was the concentration of the $\mathrm{NaOH}$ standard solution, $m$ was the mass of LPCAs in the round-bottom flask, 5 was the conversion factor, i.e., $50 \mathrm{~mL} / 10 \mathrm{~mL}$, and 45.018 was the molar weight of carboxyl.

The molecular weight of lignin was determined by gel permeation chromatography (GPC) using Agilent PL-GPC 220. The temperature of the column was $70^{\circ} \mathrm{C}$. The type of the column was PLgel. The solvent of lignin was DMF (N,N-dimethylformamide, chromatographic pure). The polymer standard used for calibration was PS (polystyrene). The calibration range was from 1000 to $10^{6}$. The software Cirrus GPC version 3.3 was used for analysis.

2.5. Characterization of RPUFs. The expansion ratio of RPUFs was the volume of RPUFs divided by the nominal initial volume of the blank RPUF. The volume of RPUFs was the volume of the spilled water when RPUFs were put into a $3000 \mathrm{~mL}$ beaker. The nominal initial volume of the blank RPUF was the sum of the volume of polyether polyol, DBTDL, surfactant, distilled water, and MDI, and the result of the sum was approximately $34.22 \mathrm{~mL}$. For each RPUF, the volume was measured at 
least 5 times to calculate the average value and the standard deviation of the expansion ratio.

The compression performance of RPUFs was characterized by a microcomputer-controlled electronic universal testing machine (Jinan Liangong Testing Technology Co. Ltd., CMT-20) with a compressive rate of $2 \mathrm{~mm} \cdot \mathrm{min}^{-1}$ according to the China National Standard GB/T 8813-2008. For each RPUF, at least 5 cubes with approximate dimensions of $2 \mathrm{~cm} \times 2 \mathrm{~cm} \times 2 \mathrm{~cm}$ were cut from it and used to calculate the average value and the standard deviation.

The water resistance test was carried out in room temperature. A sample with an approximate dimension of $2 \mathrm{~cm} \times 2 \mathrm{~cm} \times 2 \mathrm{~cm}$ was cut from each RPUF. Later, the samples were placed into distilled water for $12 \mathrm{~h}$. Afterwards, the samples were taken out and were wiped up by filter paper and then the final weight of the samples were measured. The percentage of the weight change was figured out according to the following:

$$
W=\frac{m_{2}-m_{1}}{m_{1}} \times 100 \%
$$

where $m_{1}$ was the initial weight of the sample and $m_{2}$ was the final weight of the sample.

\section{Results and Discussion}

3.1. Characterization Results of Lignin. The content of carboxyl, number-average molecular weight $\left(M_{n}\right)$, and weightaverage molecular weight $\left(M_{w}\right)$ of acid-precipitated lignin and LPCAs are shown in Table 2. After lignin was oxidized by $\mathrm{NaOCl}$ solution, the content of carboxyl increased and the molecular weight decreased, indicating that LPCAs were prepared successfully.

3.2. Effect of LPCAs on the Expansion Ratio and the Morphology of RPUFs. Table 3 shows the effect of LPCAs on the expansion ratio of RPUFs. With the loading of LPCAs increasing, the expansion ratio first increased slightly and then decreased. As dioxane was used as the solvent of LPCAs, when dioxane was added to Solution A, the viscosity of the Solution A was decreased, so that the expansion ratio of RPUFs increased when the loading of LPCAs was relatively small. When the loading of LPCAs was more than $0.53 \mathrm{wt} \%$, the expansion ratio of RPUFs was less than the blank RPUF (F0), indicating that LPCAs hindered the foaming process of RPUFs when the loading of LPCAs was relatively large.

Figure 1 is the macrophoto of RPUFs with different loadings of LPCAs. The samples in the photo are as follows: (a) F0, (b) F1, (c) F2, (d) F3, (e) F4, and (f) F5. The distance between each two adjacent black lines in each subphoto is $1 \mathrm{~mm}$. Large foam cells will result in a large volume and a large expansion ratio of the foams. The foam cells of (b) F1 and (c) F2 were larger than those of the others, which corresponded with Table 3.

3.3. Effect of LPCAs on the Compressive Strength of RPUFs. Figure 2 and Table 4 show the effect of LPCAs on the compressive strength of RPUFs. When the loading of LPCAs
TABLE 2: The titration analysis and GPC characterization results of acid-precipitated lignin and LPCAs.

\begin{tabular}{lcc}
\hline & Acid-precipitated lignin & LPCAs \\
\hline$w(\mathrm{COOH})(\%)$ & $9.02 \pm 0.03$ & $11.10 \pm 0.03$ \\
$M_{n}$ & $2.91 \times 10^{4}$ & $1.23 \times 10^{4}$ \\
$M_{w}$ & $3.48 \times 10^{4}$ & $1.25 \times 10^{4}$ \\
\hline
\end{tabular}

TABLE 3: Effect of LPCAs on the expansion ratio and the compressive strength of RPUFs.

\begin{tabular}{lcc}
\hline RPUF label & LPCA loading & Expansion ratio \\
\hline F0 & $0 \mathrm{wt} \%$ & $28.1 \pm 0.1$ \\
F1 & $0.13 \mathrm{wt} \%$ & $29.7 \pm 0.1$ \\
F2 & $0.33 \mathrm{wt} \%$ & $29.3 \pm 0.1$ \\
F3 & $0.53 \mathrm{wt} \%$ & $28.5 \pm 0.1$ \\
F4 & $0.67 \mathrm{wt} \%$ & $27.4 \pm 0.1$ \\
F5 & $1.00 \mathrm{wt} \%$ & $26.4 \pm 0.1$ \\
\hline
\end{tabular}

was between $0.53 \mathrm{wt} \%$ and $0.67 \mathrm{wt} \%$, the expansion ratio of RPUFs (e.g., F3 and F4) could be close to the blank RPUF (F0), while the compressive strength increased obviously. Particularly, when the loading of LPCAs was $0.67 \mathrm{wt} \%$, the compressive strength of RPUF was the highest and was about 59.2\% higher than F0. As a comparison, the compressive strength of a bio-based polyurethane foam with a lignin content of $10 \mathrm{wt} \%$ (based on the PEG/glycerol mixture) was about $65.8 \%$ higher than that of the blank foam [20]. Thus, although the loading of LPCAs was far less than $10 \mathrm{wt} \%$, the increasing degree of the compressive strength of the RPUF was similar to the example above, which is the highlight in this study. As LPCAs were dissolved in $80 \%(v / v)$ dioxane-water solution, the segments of LPCAs could fully stretch in the solution, which contributed to form the interactions between LPCAs and RPUFs via Van der Waals forces. LPCAs had a relatively high content of carboxyl, which increased their polarity and helped to interact with the hard and soft segments of RPUFs via hydrogen bonds. Through the interactions with RPUFs, LPCAs could increase the strength of the cell walls and then increase the compressive strength of RPUFs. Therefore, the reinforcing mechanism of LPCAs on RPUFs is similar to the reinforcing mechanism of LPCAs on waterborne polyurethane elastomers [21] and LPCAs can play a positive role of modification on RPUFs.

When the loading of LPCAs was less than $0.33 \mathrm{wt} \%$, the compressive strength of RPUFs was almost equal to F0, while the expansion ratio of RPUFs (e.g., F1 and F2) was greater than F0. When the loading of LPCAs was $0.13 \mathrm{wt} \%$, the expansion ratio of RPUF was the highest and was about $5.7 \%$ higher than F0. It is implied that if the compressive strength meets a certain requirement and the fill volume is the same, a higher expansion ratio helps to save the petroleum-derived materials of RPUFs and to lower the cost of RPUFs. For example, the China National Standard GB/T 26689-2011 Rigid polyurethane cellular plastics used in 


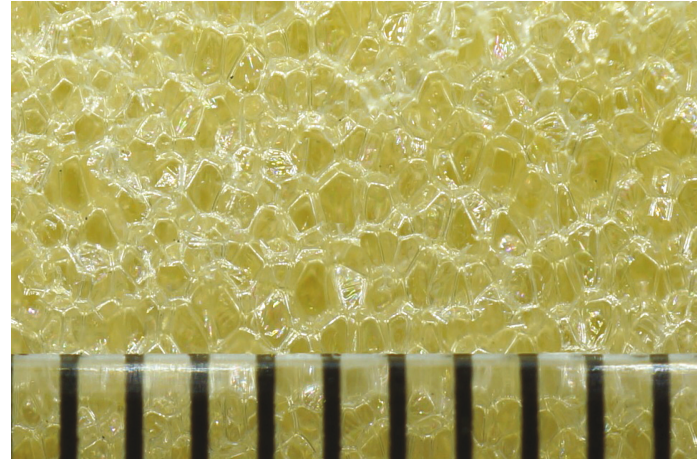

(a)

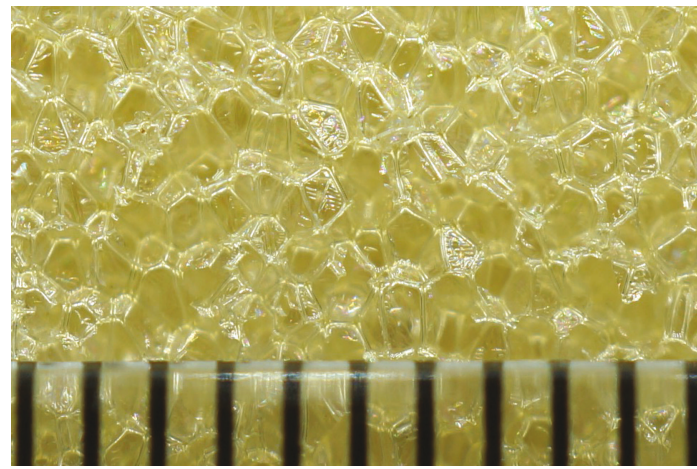

(c)

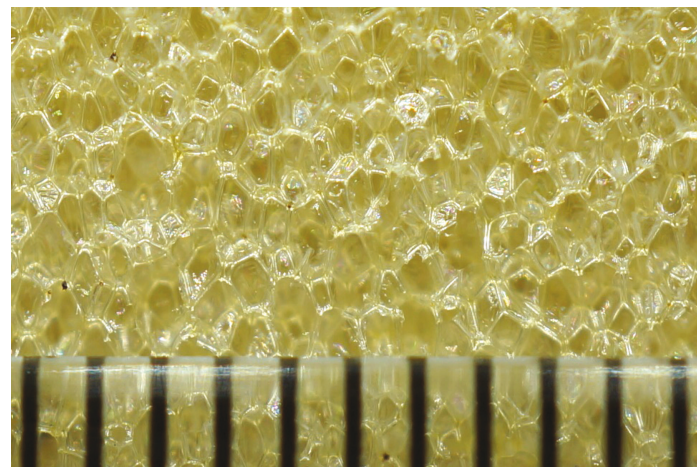

(e)

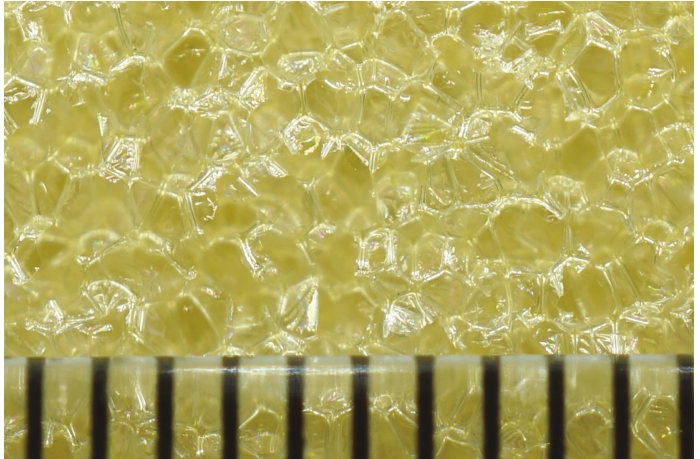

(b)

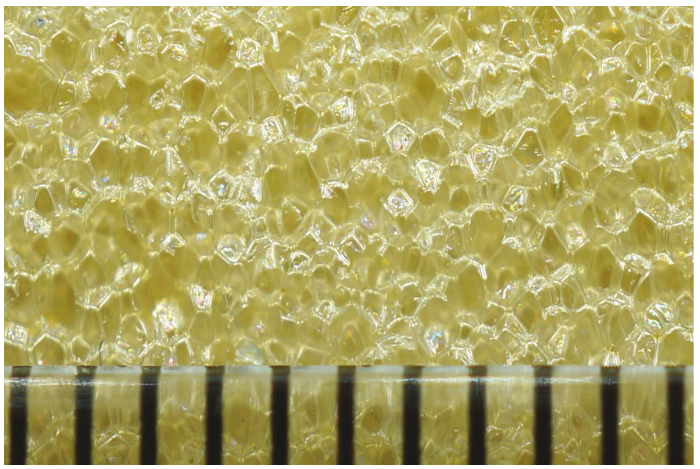

(d)

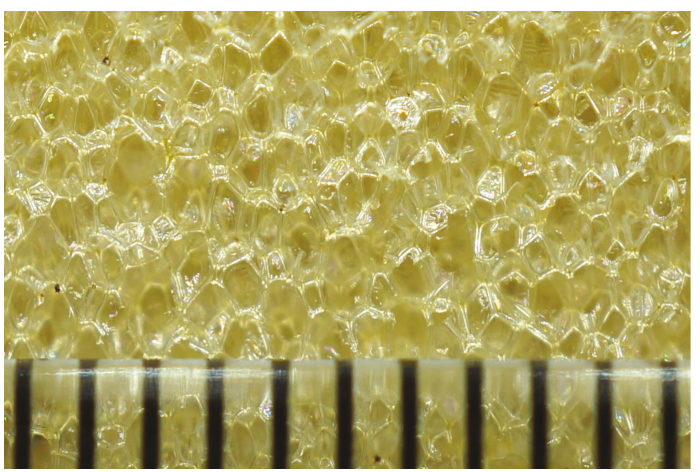

(f)

FIGURE 1: The macrophoto of RPUFs.

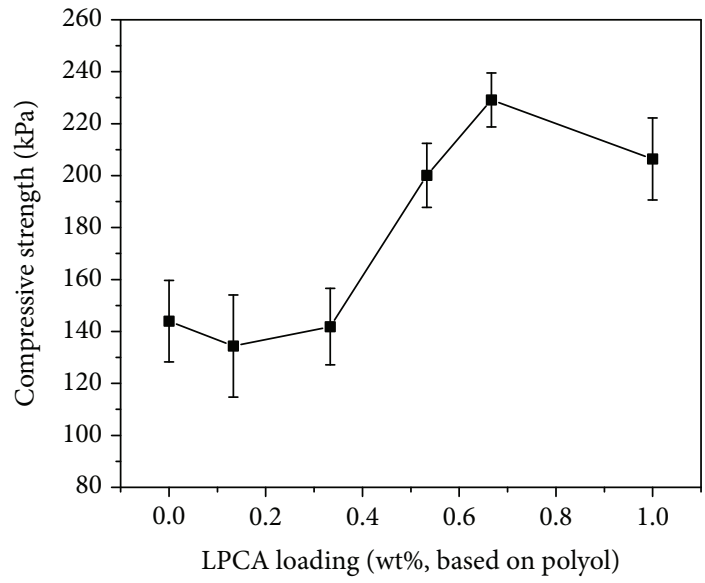

FigURE 2: Effect of LPCAs on the compressive strength of RPUFs.
TABLE 4: Effect of LPCAs on the expansion ratio and the compressive strength of RPUFs.

\begin{tabular}{lcc}
\hline RPUF label & LPCA loading & Compressive strength $(\mathrm{kPa})$ \\
\hline F0 & $0 \mathrm{wt} \%$ & $144.0 \pm 15.7$ \\
F1 & $0.13 \mathrm{wt} \%$ & $134.4 \pm 19.7$ \\
F2 & $0.33 \mathrm{wt} \%$ & $141.9 \pm 14.7$ \\
F3 & $0.53 \mathrm{wt} \%$ & $200.1 \pm 12.3$ \\
F4 & $0.67 \mathrm{wt} \%$ & $229.2 \pm 10.4$ \\
F5 & $1.00 \mathrm{wt} \%$ & $206.5 \pm 15.8$ \\
\hline
\end{tabular}

refrigerators and freezers requires the compressive strength of the type II foams which must be no less than $110 \mathrm{kPa}$. In Figure 2, the compressive strength of all RPUFs was above $110 \mathrm{kPa}$, which met the requirement of the standard. 


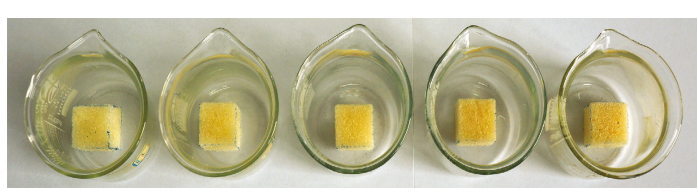

(a)

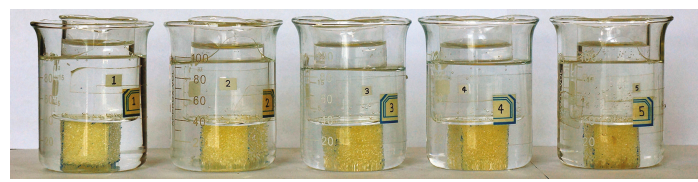

(b)

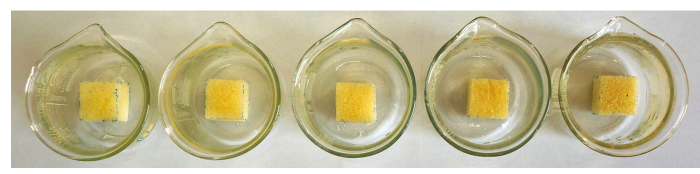

(c)

FIGURE 3: The photo of the water resistance test of RPUFs. In each subphoto, from the left to the right, the samples are F0, F1, F2, F4, and F5.

TABLE 5: The percentage of the weight change of RPUFs with different loadings of LPCAs.

\begin{tabular}{lcc}
\hline RPUF label & LPCA loading & $W$ \\
\hline F0 & $0 \mathrm{wt} \%$ & $24.3 \%$ \\
F1 & $0.13 \mathrm{wt} \%$ & $32.4 \%$ \\
F2 & $0.33 \mathrm{wt} \%$ & $41.1 \%$ \\
F4 & $0.67 \mathrm{wt} \%$ & $41.4 \%$ \\
F5 & $1.00 \mathrm{wt} \%$ & $42.1 \%$ \\
\hline
\end{tabular}

3.4. Water Resistance of RPUFs. Figure 3 shows the result of the water resistance test of RPUFs. Figure 3(a) is the photo of RPUFs which were not immersed in water. After placed in distilled water for $12 \mathrm{~h}$, the state of RPUFs is shown in Figure 3(b). Then the water in each beaker was removed and the state is shown in Figure 3(c). The percentage of the weight change of RPUFs with different loadings of LPCAs is shown in Table 5. In Figure 3(b), it can be observed that after RPUFs were immersed in water, almost no substance was dissolved from RPUFs and the foam cells of RPUFs did not collapse, indicating that the appearance of RPUFs was kept nearly the same after contacting with water for a long time. Besides, after LPCAs were introduced into RPUFs, the percentage of the weight change of RPUFs increased to different degrees. As LPCAs had a relatively high content of carboxyl, LPCAs increased the amount of polar groups of the cell walls of RPUFs, which could adsorb more water. In conclusion, the water resistance of RPUFs was not affected by LPCAs, suggesting that RPUFs with LPCAs could be used in a humid environment.

\section{Conclusion}

Lignin was oxidized to prepare LPCAs, which was then introduced into RPUFs. When the loading of LPCAs was between $0.53 \mathrm{wt} \%$ and $0.67 \mathrm{wt} \%$, the expansion ratio of RPUF could be close to the blank RPUF, while the compressive strength increased obviously. When the loading of LPCAs was $0.67 \mathrm{wt} \%$, the compressive strength of RPUF was the highest and was about $59.2 \%$ higher than that of the blank RPUF. The reinforcing mechanism of LPCAs was supposed that the interactions (e.g., Van der Waals forces and hydrogen bonds) between LPCAs and RPUFs increased the strength of the cell walls. When the loading of LPCAs was less than $0.33 \mathrm{wt} \%$, the expansion ratio of RPUFs increased slightly. Besides, the water resistance of RPUFs was not affected by LPCAs, suggesting that RPUFs with LPCAs could be used in a humid environment.

\section{Data Availability}

The data used to support the findings of this study are included within the article.

\section{Conflicts of Interest}

The authors declare that there are no conflicts of interest.

\section{Acknowledgments}

This work was partly supported by the National Natural Science Foundation of China (no. 51502108), the Foundation of Jilin Province Development and Reform Commission, China (no. 2014N145), the Science and Technology Innovation "Double Ten Project" (no. 55SS06), and the Changchun Science and Technology Bureau (no. 15SS06).

\section{References}

[1] C. Yang, L. Fischer, S. Maranda, and J. Worlitschek, "Rigid polyurethane foams incorporated with phase change materials: a state-of-the-art review and future research pathways," Energy and Buildings, vol. 87, pp. 25-36, 2015.

[2] S. H. Kim, H. C. Park, H. M. Jeong, and B. K. Kim, "Glass fiber reinforced rigid polyurethane foams," Journal of Materials Science, vol. 45, no. 10, pp. 2675-2680, 2010.

[3] K. M. Zia, H. N. Bhatti, and I. Ahmad Bhatti, "Methods for polyurethane and polyurethane composites, recycling and recovery: a review," Reactive and Functional Polymers, vol. 67, no. 8, pp. 675-692, 2007.

[4] A. Agrawal, R. Kaur, and R. S. Walia, "PU foam derived from renewable sources: perspective on properties enhancement: an overview," European Polymer Journal, vol. 95, pp. 255-274, 2017.

[5] M. S. Pawar, A. S. Kadam, B. S. Dawane, and O. S. Yemul, "Synthesis and characterization of rigid polyurethane foams from algae oil using biobased chain extenders," Polymer Bulletin, vol. 73, no. 3, pp. 727-741, 2016.

[6] Z. S. Petrović, "Polyurethanes from vegetable oils," Polymer Reviews, vol. 48, no. 1, pp. 109-155, 2008.

[7] A. Guo, I. Javni, and Z. Petrovic, "Rigid polyurethane foams based on soybean oil," Journal of Applied Polymer Science, vol. 77, no. 2, pp. 467-473, 2000.

[8] D. Kai, M. J. Tan, P. L. Chee, Y. K. Chua, Y. L. Yap, and X. J. Loh, "Towards lignin-based functional materials in a sustainable world," Green Chemistry, vol. 18, no. 5, pp. 1175-1200, 2016. 
[9] B. M. Upton and A. M. Kasko, "Strategies for the conversion of lignin to high-value polymeric materials: review and perspective," Chemical Reviews, vol. 116, no. 4, pp. 2275-2306, 2016.

[10] W. Schutyser, T. Renders, S. Van den Bosch, S. F. Koelewijn, G. T. Beckham, and B. F. Sels, "Chemicals from lignin: an interplay of lignocellulose fractionation, depolymerisation, and upgrading," Chemical Society Reviews, vol. 47, no. 3, pp. 852-908, 2018.

[11] S. H. Ghaffar and M. Fan, "Structural analysis for lignin characteristics in biomass straw," Biomass \& Bioenergy, vol. 57, no. 10, pp. 264-279, 2013.

[12] S. Laurichesse and L. Avérous, "Chemical modification of lignins: towards biobased polymers," Progress in Polymer Science, vol. 39, no. 7, pp. 1266-1290, 2014

[13] A. R. Gonçalves and P. Benar, "Hydroxymethylation and oxidation of Organosolv lignins and utilization of the products," Bioresource Technology, vol. 79, no. 2, pp. 103-111, 2001.

[14] B. L. Xue, P. L. Huang, Y. C. Sun, X. P. Li, and R. C. Sun, "Hydrolytic depolymerization of corncob lignin in the view of a bio-based rigid polyurethane foam synthesis," RSC Advances, vol. 7, no. 10, pp. 6123-6130, 2017.

[15] H. Lange, S. Decina, and C. Crestini, "Oxidative upgrade of lignin - recent routes reviewed," European Polymer Journal, vol. 49, no. 6, pp. 1151-1173, 2013.

[16] C. Crestini, M. Crucianelli, M. Orlandi, and R. Saladino, "Oxidative strategies in lignin chemistry: a new environmental friendly approach for the functionalisation of lignin and lignocellulosic fibers," Catalysis Today, vol. 156, no. 1-2, pp. 8-22, 2010.

[17] A. Kalliola, T. Vehmas, T. Liitiä, and T. Tamminen, "Alkali- $\mathrm{O}_{2}$ oxidized lignin - a bio-based concrete plasticizer," Industrial Crops and Products, vol. 74, pp. 150-157, 2015.

[18] N. Mahmood, Z. Yuan, J. Schmidt, and C. (. C.). Xu, “Depolymerization of lignins and their applications for the preparation of polyols and rigid polyurethane foams: a review," Renewable and Sustainable Energy Reviews, vol. 60, pp. 317-329, 2016.

[19] S. X. Ren, G. Q. Jiang, and H. J. Qu, Tutorials on Chemical Experiments of Plant Fibers, Northeast Forestry University Press, Harbin, China, 2008.

[20] X. Huang, C. F. de Hoop, J. Xie, C.-Y. Hse, J. Qi, and T. Hu, "Characterization of biobased polyurethane foams employing lignin fractionated from microwave liquefied switchgrass," International Journal of Polymer Science, vol. 2017, Article ID 4207367, 8 pages, 2017.

[21] R. Wang, B. Zhou, Y. Zhu, and Z. Wang, "Effects of ligninderived polycarboxylic acids on the properties of waterborne polyurethane elastomers," International Journal of Polymer Science, vol. 2018, Article ID 7989367, 7 pages, 2018. 


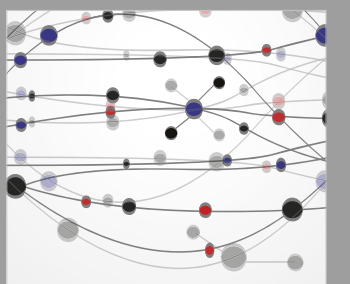

The Scientific World Journal
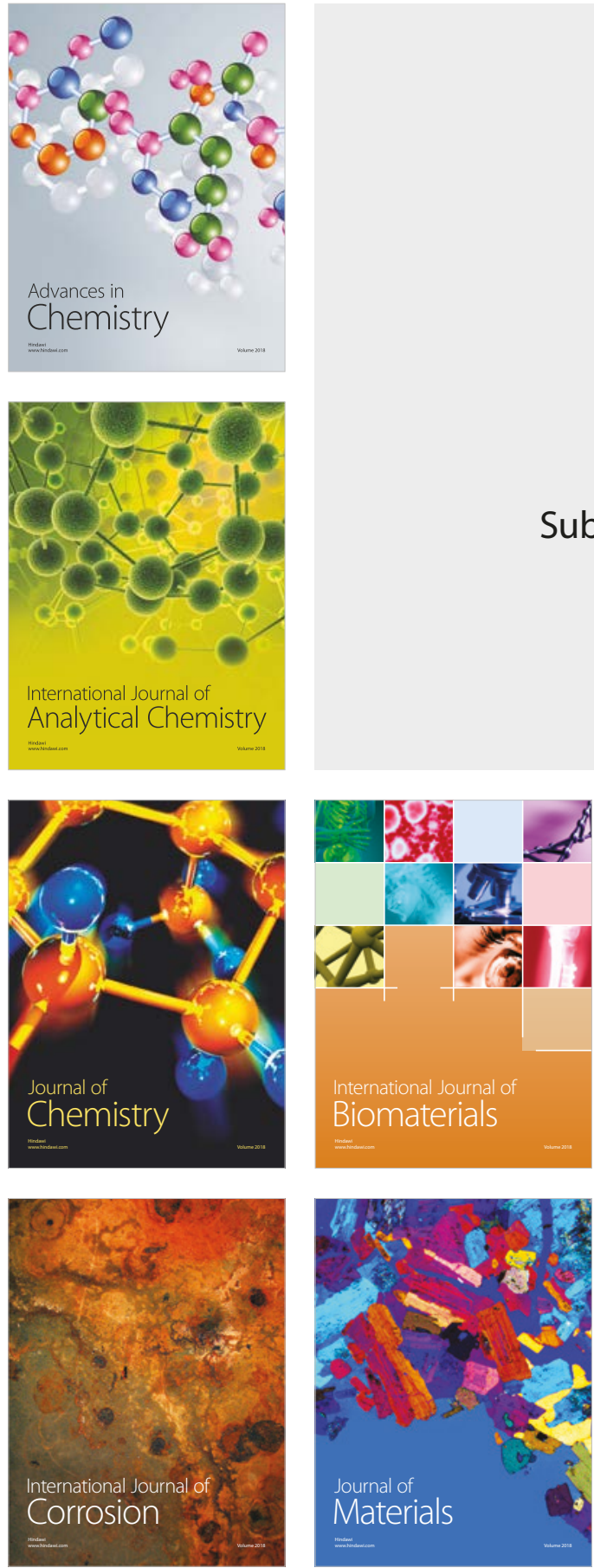

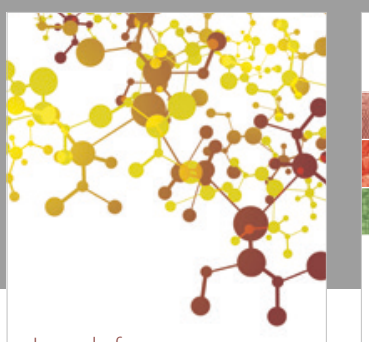

Journal of

Applied Chemistry
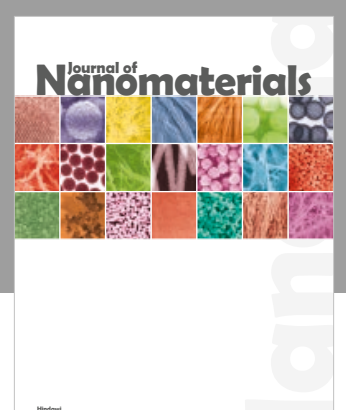

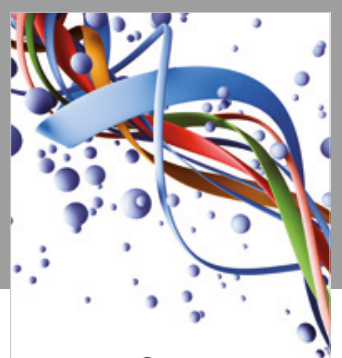

Scientifica

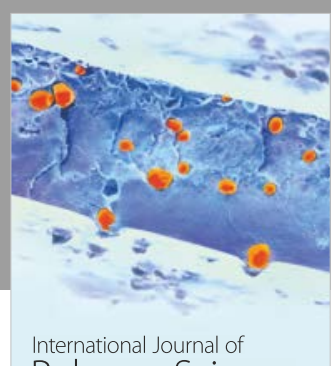

Polymer Science

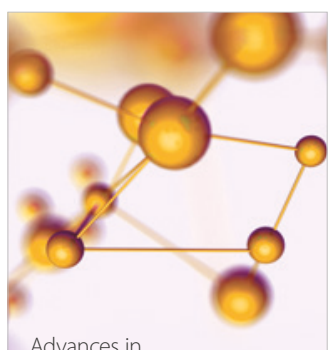

Physical Chemistry
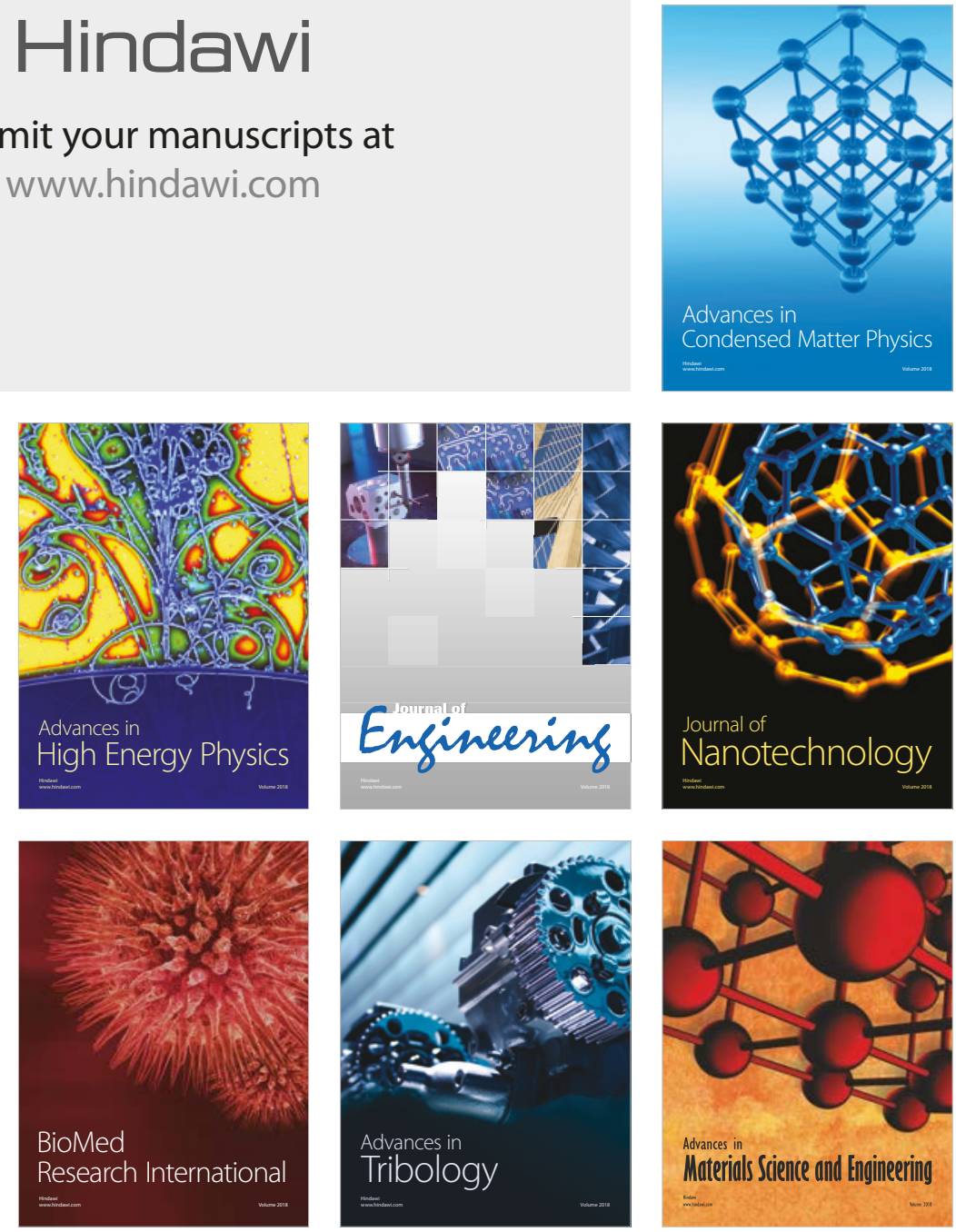